\title{
Research on Precipitate Behavior during Holding Process of X80 Pipeline Steel
}

\author{
Tao Niu ${ }^{1, a,}$, Chenggang $\mathrm{An}^{1, \mathrm{~b}}$, Yongwen Jiang ${ }^{1, \mathrm{c}}$, Xinlang Wu ${ }^{2, \mathrm{~d}}$, Caixia Zhang ${ }^{2, \mathrm{e}}$, Chen \\ $\mathrm{Yu}^{2, \mathrm{f}}$, Xiaoli Dai, ${ }^{1, \mathrm{~g}}$ \\ ${ }^{1}$ Shougang Research Institute of Technology, Beijing 100043, China \\ ${ }^{2}$ Shougang Qian'an Iron \&Steel Co.Ltd, Qian'an 064404, China \\ aJohnniu@126.com, banchenggang@shougang.com.cn, ${ }^{C}$ jiangyongwen@shougang.com.cn \\ dwuxinlang@sgqg.com.cn, ${ }^{\mathrm{e}}$ zhangcaixia@sgqg.com.cn, yuchen@sgqg.com.cn, \\ gdaixiaoli1302@sina.com
}

\begin{abstract}
PTT (Precipitate-Temperature-Time) curve of X80 pipeline steel was obtained by strain relaxation method. The evolution of precipitate particle size during holding process was simulated combined with kinetic calculation, and observed using TEM in the samples of industrial produced X80 steel with different holding time. It is revealed that the shape of PPT curve is typical "C" type with the nose temperature of $900{ }^{\circ} \mathrm{C}$ and incubation time of about $5 \mathrm{~s}$. Kinetic calculation results show that the average particle size increases obviously with the increment of holding time. Meantime, after holding for $90 \mathrm{~s}$ at $950^{\circ} \mathrm{C}$, observation of industrial produced X80 steel reveals that the proportion of precipitate particles larger than $60 \mathrm{~nm}$ increases dramatically, which basically agreed with the calculation results. Dissolved $\mathrm{Nb}$ can effectively reduce grain boundary mobility and retard recrystallization by solute drag effect. Therefore, it is strongly recommended to shorten the holding time without increasing holding temperature in industrial production, so that to reduce precipitate of $\mathrm{Nb}$ at high temperature, and increase the strength and toughness of steel.
\end{abstract}

\section{Introduction}

Pipeline is the most cost-effective way in oil and gas transportation over long distances. With the increasing requirement of flow capacity, higher pressure and larger diameter pipe transportation is more and more widely used to save the costs [1], which brings higher demands of strength and toughness for the pipeline steel. The mechanical properties of X80 pipeline steel are determined by combined characteristics, such as composition, TMCP technology, microstructure, as well as precipitates[2]. Precipitation is well known as one of the most important strengthening mechanisms for pipeline steels. Fine carbonitride particles induced by hot deformation, taking $\mathrm{NbC}$ for instance, can dramatically retard the recrystallization and thereby refine austenite grains. Precipitates that formed during laminar cooling also play an important role in significantly enhancing the strength of pipeline steels. Although some investigations have already been put forward on

*Corresponding author:Johnniu@126.com 
precipitation characteristic of final pipeline steels product for different grades [3,4], as well as the effect of precipitate on the austenitization and austenite grain growth during reheating process [5], few studies focusing on the precipitation behavior and its effect during holding process between roughing and finishing rolling have been reported.

In the present work, the precipitation behavior of X80 pipeline steel during holding process between roughing and finishing rolling was investigated. PTT curve was tested using strain relaxing experiment, based on which, the particle size revolution was simulated combined with dynamic calculation, hoping it can provide some theoretical foundations for TMCP technology design for X80 pipeline steel.

\section{Materials and Experimental Procedure}

\subsection{Experimental Materials}

X80 pipeline steel used in this work were sampled from two hot rolling coils with different holding time between roughing and finishing rolling. The main compositions include $0.055 \mathrm{C} ; 0.2 \mathrm{Si} ; 1.85 \mathrm{Mn} ; 0.085 \mathrm{Nb}$, as well as $\mathrm{Ti}, \mathrm{Mo}, \mathrm{Cr}$ and so on (wt\%). The production flow is converter - LF - $\mathrm{RH}$ - continuous casting - two-stage themo-mechanical processing and laminar cooling. The thickness of slab is about $230 \mathrm{~mm}$ and the final coil is about $16.5 \mathrm{~mm}$ (thickness)* $1550 \mathrm{~mm}$ (width).

\subsection{PTT Experimental Procedure}

PTT experiment was put forward on Gleeble-1500 thermo-simulator. The size of initial specimen is about $\varphi 8^{*} 12 \mathrm{~mm}$. Firstly, the specimen was reheating to $1200^{\circ} \mathrm{C}$ in rate of $10^{\circ} \mathrm{C} / \mathrm{s}$ and soaked for $25 \mathrm{~min}$ in order to guarantee the complete solution of alloys. Then they were cooled to certain temperature in rate of $1.5^{\circ} \mathrm{C} / \mathrm{s}$ and compressed after held for $60 \mathrm{~s}$. The constant strain was kept for 1000s under the deformation temperature, and the variation of stress was tested and record. The deformation temperatures used in this work include $800^{\circ} \mathrm{C}, 850^{\circ} \mathrm{C}, 900^{\circ} \mathrm{C}$, and $950^{\circ} \mathrm{C}$. The deformation is $20 \%$ with rate of $5 / \mathrm{s}$. Logarithmic time to stress curve (logt-Stress) was made using the experiment results, on which, the initial and finishing position of the stress plateau represent the starting and finishing time of precipitate, respectively.

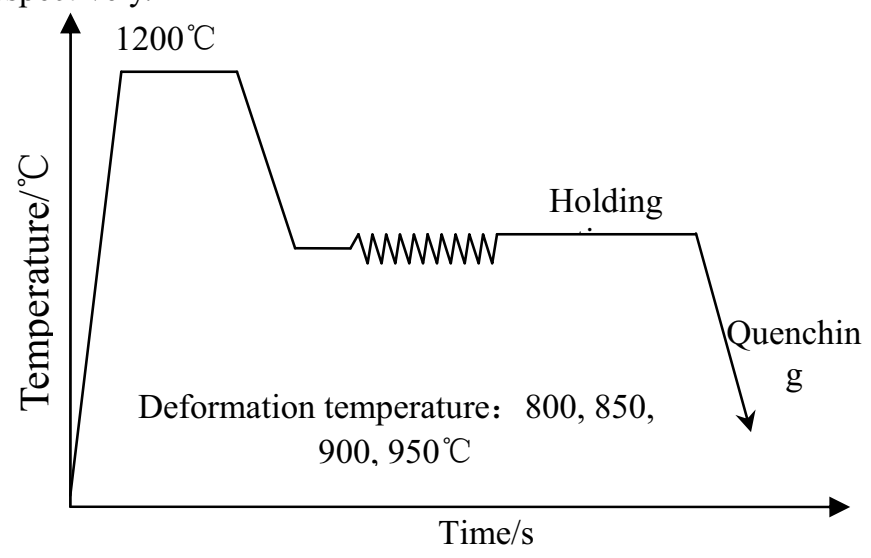

Fig.1 Process of strain relaxation experiment 


\subsection{Precipitate Behavior Analysis}

In order to analyze the precipitate particle size evolution during holding process, X80 samples were taken with different holding time. Carbon extraction replicas was made according to relative standard and the morphology and distribution of precipitate particles was observed using JEM-2100F (HR) TEM.

\section{Experiment results and analysis}

\subsection{PTT experiment results}

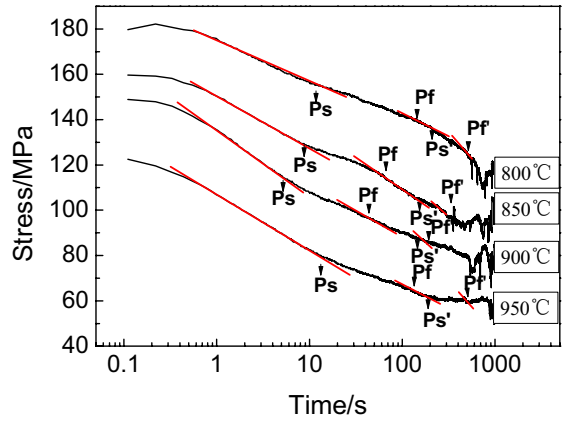

Fig.2 Strain relaxation curve at different relaxation temperatures

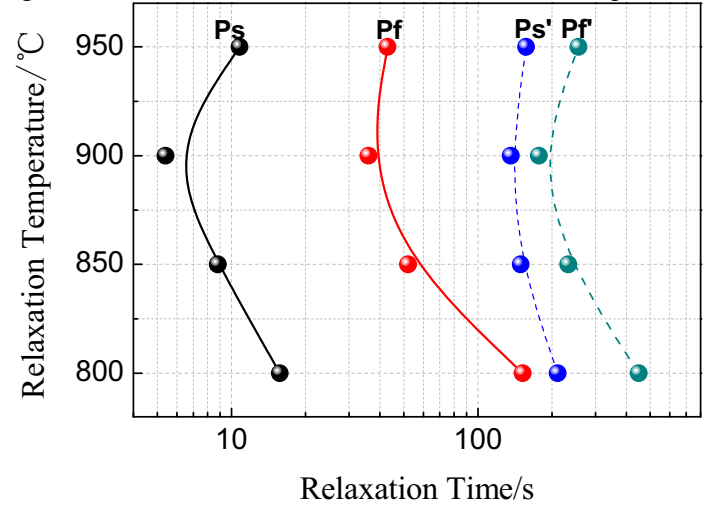

Fig.3 PTT curve

The stress relaxation curves for the two steels at different temperatures are shown in Fig. 2. $P_{\mathrm{s}}, P_{\mathrm{f}}$ represent the starting and finishing time of precipitate, respectively. And for the second time precipitate, the time was indicated as $P^{\prime}{ }_{\mathrm{s}}$, and $P^{\prime}{ }_{\mathrm{f}}$.

It can be concluded that the PTT curve is typical "C" shape. It is because the precipitate process of $\mathrm{Nb} / \mathrm{Ti}$ is a nucleation and growth process controlled by diffusion, including diffusion speed in austenite, as well as the degree of super-saturation of solute atom [6]. When the temperature is higher, the diffusion speed is fast, while the supersaturated chemical driving force of solute atom in austenite reduces. Therefore, the precipitate process is slow down. On the other hand, although the supersaturated chemical driving force can increase when the temper goes down, the diffusion speed also decreased and slower the precipitate process at the end. It can be concluded that there must be a certain temperature with both proper supersaturated chemical driving force and atom diffusion rate, when the precipitate speed reaches the fastest. This temperature is always defined as the nosing temperature of PTT curve. For experimental material used in this work, this nosing temperature is about $900^{\circ} \mathrm{C}$, and the incubation time at this temperature is about $5 \mathrm{~s}$. 
In addition, there is obvious phenomenon of secondary precipitate. The reason of precipitate more than once is because within a period of time, only the solute atoms near the high density of location get the chance to precipitate fast. Thus the surrounding depleted regions of solute atom forms and retards the following precipitate. Dislocations unevenly distribute in the microstructure, and some of them get depinning during relaxation due to thermal activation. If these dislocations move to the regions with less precipitates from high density region, it is equivalent that they moved to the new regions with high density of solute atom, where they may become the new preferred nucleation position of precipitate and get pinned again. Therefore, the secondary precipitate can start only when the dislocations get out from the pinning of the particles precipitated before. Besides, the dislocation density reduces on some level at this time, so it takes more time for precipitation to start again.

It also should be noticed that the investigation of strain relaxation experiment was carried out after only one pass of deformation. As for the industrial production, the great effect of deformation on deformation induced precipitate cannot be ignored, and the nosing temperature will be rose up.

\section{Dynamics Calculation of Precipitate Particle Size}

\subsection{Kinetic Model}

During the precipitation transformation of micro-alloy elements carbonitride, the composition of precipitate is totally different from that of the matrix. The growth of precipitate particles is controlled by long distance diffusion of solute atom. Therefore, the growth speed of new phase crystal nucleus mainly depends on the diffusion speed of solute atom. According to the investigation before [6], for the precipitation transformation process, during which the nucleation ratio attenuated to zero rapidly, all the nucleation mainly forms at time of $t_{\mathrm{s}}$. In this way, when the phase transformation ends the average particle size of the new phase in system $\bar{R}_{\mathrm{f}}$ will be:

$$
\begin{gathered}
\bar{R}_{\mathrm{f}}=\lambda D^{1 / 2}\left(t_{\mathrm{f}}-t_{\mathrm{s}}\right)^{1 / 2} \\
\lambda=(-k)^{1 / 2}
\end{gathered}
$$

Where, $D$ stands for solute atom diffusion coefficient in matrix. $\lambda$ is related to saturation function $k$, which is defined as:

$$
k=-\frac{2\left(c_{0}-c_{\mathrm{M}}\right)}{c_{\mathrm{N}}-c_{\mathrm{M}}}
$$

Where, $c_{0}$ is average solute atom concentration in matrix, while $c_{\mathrm{M}}$ and $c_{\mathrm{N}}$ stand for solute atom concentration in matrix and precipitate on the boundary of phase interface, respectively. 


\subsection{Dynamics Calculation Results}

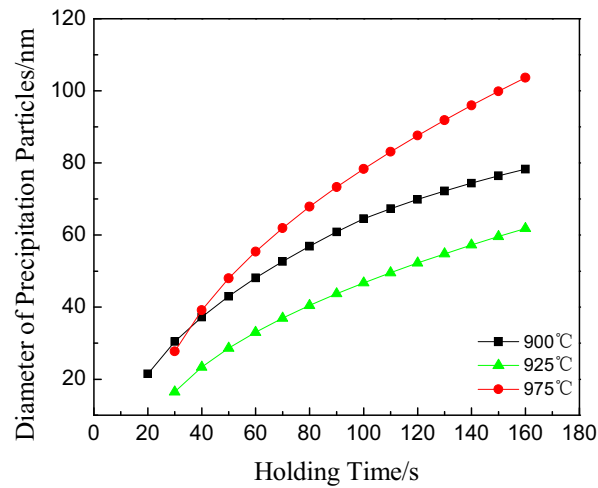

Fig.4 The relationship between holding time and diameter of precipitation particles

Calculation result of precipitate particle dimensions obtained after different holding time is shown in Fig. 4. As the holding temperature rises, average particle size increases obviously after holding for the same time. After $40 \mathrm{~s}$, the average size of precipitate particles reaches to $23 \mathrm{~nm} 3,37 \mathrm{~nm}$, and $9 \mathrm{~nm}$ for $925^{\circ} \mathrm{C}, 950^{\circ} \mathrm{C}$ and $975^{\circ} \mathrm{C}$, respectively. And the dimension also increases with the increment of holding time. After holding for $90 \mathrm{~s}$, the average dimension will reach to $44 \mathrm{~nm}, 60 \mathrm{~nm}$ and $73 \mathrm{~nm}$.

It also should be noticed that during the actual deformation of rolling, large quantities of dislocation will experience the evolution of configurations as the holding time lasts. In fact, after holding for $200 \mathrm{~s}$, over-aging behavior might occur and the precipitate particles start to gather and grow coarse, which will reduce the retardation effect to grain boundaries and lead to the grain coarsening ${ }^{[7]}$.

\subsection{Precipitate behavior during actual production}

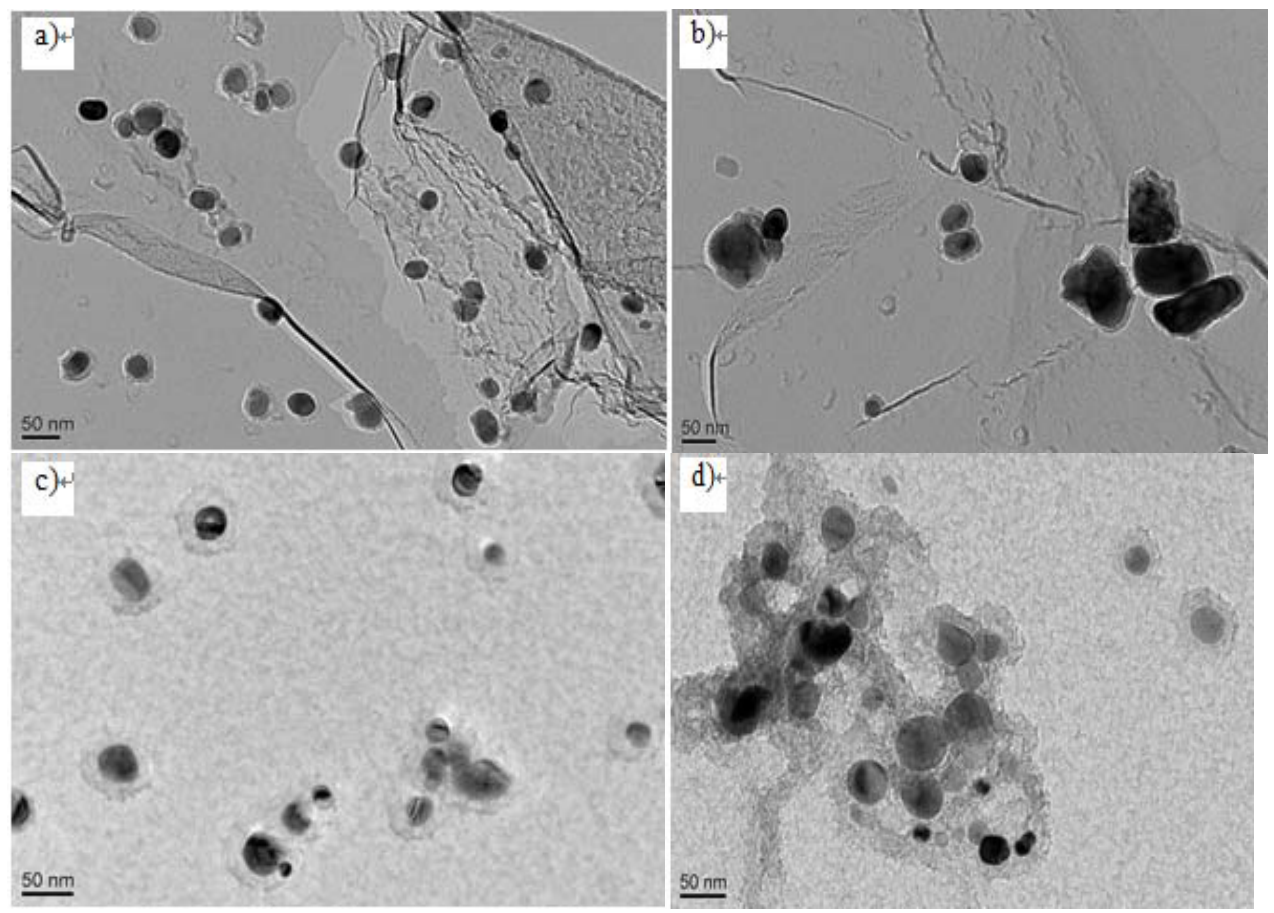




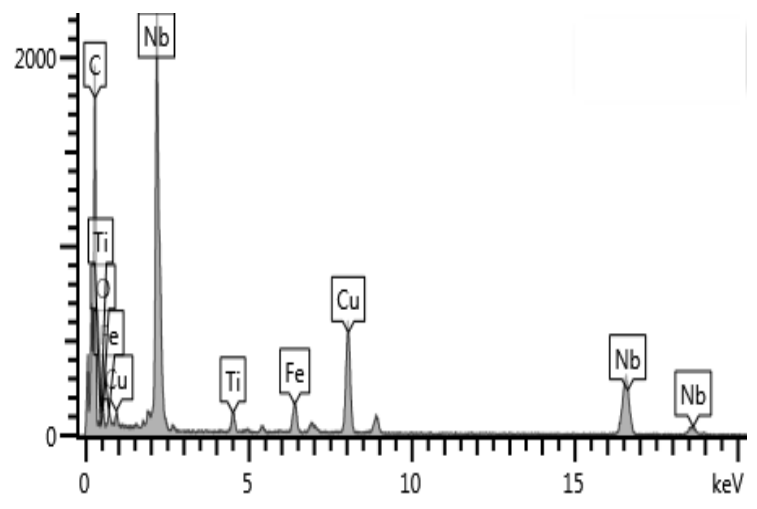

Fig. 5 Morphology and composition of precipitation particles with different holding time at $950^{\circ} \mathrm{C}$

a,c) Holding time: 40s; b,d) Holding time : 90s; e) Typical spectrum

During the actual production of X80 pipeline steel, the finishing temperature of roughing rolling is about $950^{\circ} \mathrm{C}$. Carbon replica extraction was employed in order to observe precipitate particle size and distribution obtained after different holding time, as is shown in Fig. 5. According to the comparison, it is revealed that when holding for about 40 s, most of the particles grow up to $20 \sim 50 \mathrm{~nm}$, and the distribution is uniform (Fig. 5, a, c). However, with the increment of holding time to $90 \mathrm{~s}$, the proportion of particles larger than $60 \mathrm{~nm}$ rises dramatically, while that of particles between $30 \sim 60 \mathrm{~nm}$ drops (Fig. 5, b, d), which confirms the dynamic calculation results above. According to the energy spectrum analysis (Fig. 5 e), the particles are mainly typical $\mathrm{Nb}(\mathrm{C}, \mathrm{N})$.

\section{Analysis and Discussion}

X80 pipeline steel investigated in this study employed a typical low $\mathrm{C}$ high $\mathrm{Nb}$ composition system. Before precipitate as $\mathrm{NbC}$, the solid dissolved $\mathrm{Nb}$ plays an important part in decreasing the grain boundary mobility by solution drag effect, so that to retard static recrystallization effectively. Past research has shown that in order to guarantee the solution drag effect, sufficient residual solid dissolved $\mathrm{Nb}$ should stay in the matrix, of which the typical range is $0.03 \sim 0.04 \%$. This part of residual solid dissolved $\mathrm{Nb}$ contributes to the grain refinement during the following cooling process and further improve the strength [8]. The effect of solid dissolved $\mathrm{Nb}$ on grain boundary mobility can be described using slow branch model in Cahn's theory [9] as:

$$
M(N b, T)=\left(\frac{1}{M_{\text {pure }}}+\alpha \cdot X_{N b}\right)^{-1}
$$

Where, the coefficient of $\mathrm{Nb} \alpha$ can be expressed as:

$$
\alpha=\frac{N v(k T)^{2}}{\delta E_{0} D_{N b}^{I n t}}\left[\sinh \left(\frac{E_{0}}{k T}\right)-\frac{E_{0}}{k T}\right]
$$

Where, $\mathrm{K}$ stands for boltzmann's constant, $\mathrm{T}$ is absolute temperature, $N v$ is atom number in unit volume, $\delta$ is grain boundary with, usually taken as $1 \sim 2 \mathrm{~nm}$. $D_{N b}^{\text {Int }}$ is diffusion coefficient of $\mathrm{Nb}$ across grain boundary, which can be calculated using the formula below [10]: 


$$
\begin{gathered}
D_{N b}=0.001825 \exp (-30910 / T) \\
D_{N b}^{I n t}=10 D_{N b}
\end{gathered}
$$

$E_{0}$ stands for segregation energy of solute atom on the boundary, and is always taken as $0.2 \mathrm{eV}$ according to the past investigations [8].

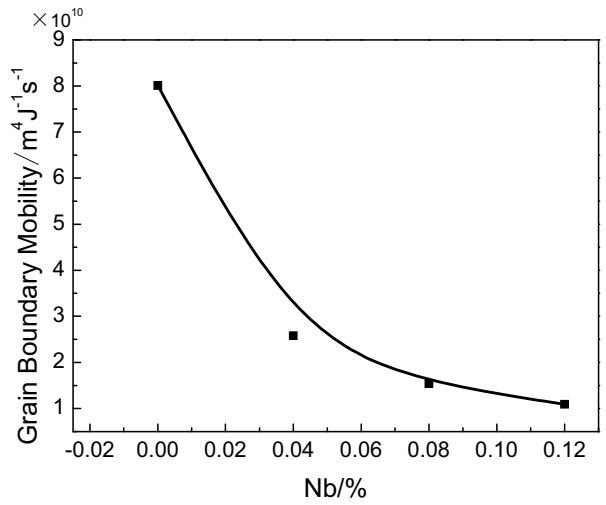

Fig.6 Relationship between dissolved $\mathrm{Nb}$ and grain boundary mobility

The effect of solute dissolved $\mathrm{Nb}$ on grain boundary mobility in shown in Fig. 6. At the same temperature, the grain boundary mobility decreases with the addition of $\mathrm{Nb}$. As the solute dissolved $\mathrm{Nb}$ content rises from 0 to $0.12 \%$, the grain boundary mobility drops from $8.01131 \times 10^{-10} \mathrm{~m}^{4} \cdot \mathrm{J}^{-1} \cdot \mathrm{s}^{-1}$ to $1.09326 \times 10^{-10} \mathrm{~m}^{4} \cdot \mathrm{J}^{-1} \cdot \mathrm{s}^{-1}$, which illustrates that solute dissolved $\mathrm{Nb}$ content has an obvious influence on grain boundary mobility.

Despite of the effect on recrystallization, solute dissolved $\mathrm{Nb}$ also plays an important role in retarding phase transformation and refining grain size. It can precipitate as smaller $\mathrm{NbC}$ particles in ferrite and contribute to strength and toughness by precipitation strengthening effect as well. Therefore, it can reduce the precipitate of $\mathrm{Nb}$ at higher temperature during holding process by shorten holding time properly based on the lower holding temperature. In this way, the solute dissolved $\mathrm{Nb}$ content gets improved, and contributes more to control recrystallization during finishing rolling process, retard of phase transformation, and increase the effective precipitate in ferrite, which finally guarantees to achieve the refined acicular ferrite microstructure and strength-toughness.

\section{Conclusions}

1) Stress relaxation experiment reveals that the PPT curve of $\mathrm{X} 80$ pipeline steel in this study is typical " $\mathrm{C}$ " type with the nose temperature of $900^{\circ} \mathrm{C}$ and incubation time of about $5 \mathrm{~s}$. There is also typical second precipitate phenomenon.

2) Kinetic calculation results show that the average particle size increases obviously with the increment of holding time. After holding for $90 \mathrm{~s}$, the average dimension will reach to $44 \mathrm{~nm}, 60 \mathrm{~nm}$ and $73 \mathrm{~nm}$ for $925^{\circ} \mathrm{C}, 950^{\circ} \mathrm{C}$ and $975^{\circ} \mathrm{C}$, respectively.

3) Meantime, after holding for $90 \mathrm{~s}$ at $950^{\circ} \mathrm{C}$, observation of industrial produced $\mathrm{X} 80$ steel reveals that the proportion of precipitate particles larger than $60 \mathrm{~nm}$ increases dramatically, which basically match with the calculation results.

4) Dissolved $\mathrm{Nb}$ can effectively reduce grain boundary mobility and retard recrystallization by solute drag effect. Therefore, it is strongly recommended to shorten the holding time without increasing holding temperature in industrial 
production, so that to reduce precipitate of $\mathrm{Nb}$ at high temperature, and increase the strength and toughness of steel.

\section{References}

1. B Tanguya, T T Luu, G Perrinb, A Pineau, J Besson. Plastic and damage behavior of a high strength X100 pipeline steel: Experiments and modeling. International Journal of Pressure Vessels and Piping, 85(2008): 322 335

2. S Shanmugam, N K Ramisetti, R D K Misra. Microstructure and high strength-toughness combination of a new $700 \mathrm{MPa}$ Nb-microalloyed pipeline steel. Materials Science and Engineering, 478 (2008): 26 37

3. H Yu, Y Sun, Q X Chen, H T Jihang, L H Zhang. Precipitation behaviors of X70 acicular ferrite pipeline steel [J]. Journal of University of Science and Technology Beijing, 13(2006): 523 527

4. T Niu, Y L Kang, H W Gu, Y Q Yin, M L Qiao. Precipitation Behavior and Its Strengthening Effect of X100 Pipeline Steel [J]. Journal of Iron And Steel Research, International, 17(2010): 73 78

5. Q Y Liu, X J Sun, S J Jia, L LZ hang, G J Huang, Y Ren. Austenitization Behaviors of X80 Pipeline Steel with High $\mathrm{Nb}$ and Trace Ti Treatment [J]. Journal of Iron And Steel Research, International, 16(2009): 58 62

6. Q L Yong. Second phase in iron and steel[M]. Metallurgical Industry Press, Beijing, 2006

7. S Q Yuan, S W Yang, H B Wu, et al. Precipitation Behavior During Relaxation After Deformation and Aging in Microalloy Steel Containing $\mathrm{Nb}[\mathrm{J}]$, Heat Treatment of Metals, 30(2005): 24-27.

8. H S Zurob, G Zhu, S V Subramanian, et al. Analysis of the effect of Mn on the recrystallization kinetics of high $\mathrm{Nb}$ steel: an example of physically-based alloy design[J]. ISIJ International, 45(2005): 713-722.

9. J W Cahn. The Impurity-drag effect in grain boundary motion. Acta Mater, 10(1962): 789-798.

10. S Masayoshi. An Analysis of the solute drag effect of $\mathrm{Nb}$ on recrystallization of ultra-low carbon steel [J]. ISIJ International, 38(1998):47-552. 